\title{
Microfluidic effects of transporting signaling components in cell coculture chips
}

\author{
Guoqing Hu $\cdot$ Dongqing Li
}

Received: 2 February 2008/Accepted: 7 April 2008/Published online: 27 May 2008

(c) Springer-Verlag 2008

\begin{abstract}
A theoretical model has been developed to investigate the microfluidic transport of the signaling chemicals in the cell coculture chips. Using an epidermal growth factor (EGF)-like growth factor as the sample chemical, the effects of velocities and channel geometry were studied for the continuous-flow microchannel bioreactors. It is found that different perfusion velocities must be applied in the parallel channels to facilitate the communication, i.e., transport of the signaling component, between the coculture channels. Such communication occurs in a unidirectional way because the signaling chemicals can only flow from the high velocity area to the low velocity area. Moreover, the effect of the transport of the signaling component between the coculture channels on the growth of the monolayer cells and the multicellular tumor spheroid (MTS) in the continuous-flow coculture environment were simulated using 3D models. The numerical results demonstrated that the concentration gradients will induce the heterogeneous growth of the cells and the MTSs, which should be taken into account in designing the continuous-flow perfusion bioreactor for the cell coculture research.
\end{abstract}

Keywords Microfluidics - Cell coculture · Numerical simulation $\cdot$ Concentration gradients

\section{G. $\mathrm{Hu}$}

LNM, Institute of Mechanics, Chinese Academy of Sciences, 100080 Beijing, China

\section{Li (ه)}

Department of Mechanical Engineering, Vanderbilt University, VU Station B 351592, 2301 Vanderbilt Place, Nashville,

TN 37235-1592, USA

e-mail: dongqing.li@vanderbilt.edu

\section{Introduction}

Cell culture is a key step in cell biology, biotechnology, tissue engineering, and clinic application. Although the traditional in vitro culture techniques are widely used in laboratory experiments, it is questionable that cell growth in vitro is identical to that in vivo as the conventional in vitro cell culture methods grow cells in a homogeneous static and macroscale environment such as polystyrene dishes or wells. Recent development of microfabrication and microfluidic technology has brought the exciting potential applications for cell culture and cellular-scale (around several micron) study (Anderson and van den Berg 2004; El-Ali et al. 2006). Contrast to the conventional culture methods, microfluidic cell culture systems can create small and complex architecture to mimic the in vivo environment for cell growth (Walker et al. 2004). The nutrients can be more efficiently delivered to the cells through a continuous-flow perfusion in the microfluidic bioreactors. Several microscale culture systems, mainly using silicon (Martinoia et al. 1999; Powers et al. 2002; Pantoja et al. 2004) and silicone elastomer (PDMS) (Folch and Toner 1998; Walker et al. 2002; Leclerc et al. 2003; Tourovskaia et al. 2005), have been designed for culture of different cells. These microfluidic chips offer the advantages of better control of culture conditions, improved throughput, and more capability to address phenotypic responses at the cellular length scale.

A living organ normally consists of multiple cell types to perform physiological roles. Communication between cells in a multicellular environment can be classified into three signaling types: autocrine (self-stimulating) (Sporn and Todaro 1980; Lauffenburger et al. 1995), paracrine (stimulating nearby cells) (Finch et al. 1989; Klein et al. 1989), and endocrine (stimulating cells in remote tissues) (Powers 
et al. 2000; Ray 2007). There have been many efforts towards identifying these complex interactions between cells. Most conventional studies try to examine the multicelluar effects by transferring the conditioned culture media from one group of cells to another (Ellington et al. 1990; Thio et al. 1993). Other researchers use polymer porous membrane to separate different cell types in the shared media environment (Fillinger et al. 1993; Attawia et al. 1999). Using various microfabrication and microfluidic technique, different cells can be deposited on different locations within the coculture microdevices. Examples of microfluidic cell coculture platform include microarray for micro-patterned hepatocyte cells with fibroblast cells (Kane et al. 2006), multilayer coculture for biomimmetic blood vessels (Tan and Desai 2004), and patterned substrate for selective attachment and growth of several cell types (Rhee et al. 2005). Wei et al. (2006) designed a microfluidic system to evaluate the interaction between cocultured cell types, by distributing cytokines expressed in up-stream wells and then generating a concentration gradient in downstream wells. More Recently, Irimia et al. (2007) developed a microfluidic device with co-running and connection microchannels to study the chemotaxis of the HL-60 cells. They used a contact zone to compensate the unbalanced flow rates within the co-running microchannels.

Experiments are the major method in understanding biological mechanisms. Experiments, however, are timeconsuming, expensive, and sometimes difficult to control. Numerical modeling can help researchers to determine rapidly the operating conditions and thus reduce the number of experiments required. Several models have been already developed to study the cell culture (Roy et al. 2001; Williams et al. 2002; Mehta and Linderman 2006; Hu and Li 2007; Hu et al. 2007).

This study presented a theoretical model and the numerical study of a conceptual microfluidic chip for cell coculture. The basic configuration has two or more separate main microfluidic channels connected by narrow microchannels, as illustrated in Fig. 1. A similar microfluidic configuration has been used to study the growth of the central nervous system axons (Taylor et al. 2005). In their experiments, microgrooves between the main culture compartments were implemented to isolate axons and direct the axongrowth. In our design, the narrow connection microchannel is small enough so that it physically isolates different cell types in the main channels while still allows the cell communication via convection and diffusion of the culture medium. Different from conventional cell culture methods and other static diffusion-only microfluidic culture systems, we used continuous-flow perfusion because it is more efficient in delivering nutrients. Unlike the work of Irimia et al. (2007), we investigated how the signaling component transports between the main channels and its effects on the cell growth under different coculture conditions. The proposed microfluidic system is capable of controlling the delivery direction of the signaling component, by adjusting the flow rates in the co-running microchannels, compared to the previous work (Wei et al. 2006). The transportation of an epidermal growth factor (EGF)-like type of growth factor, as the model of endocrine signaling process, was investigated for various flow rates and microchannel sizes. Finally, the effects of the growth factor concentration gradients on the monolayer cells and the multicellular tumor spheroid (MTS) were studied.

\section{Theoretical and numerical models}

Figure 1 shows schematically the basic geometry of the microfluidic coculture bioreactor. It consists of two main culture microchannels, with a narrow connection channel in-between. $L$ and $W$ are the length and width of the main culture channel while $l$ and $w$ represent the length and width of the narrow connection channel. The origin of the coordinates is located at the left bottom corner of the left

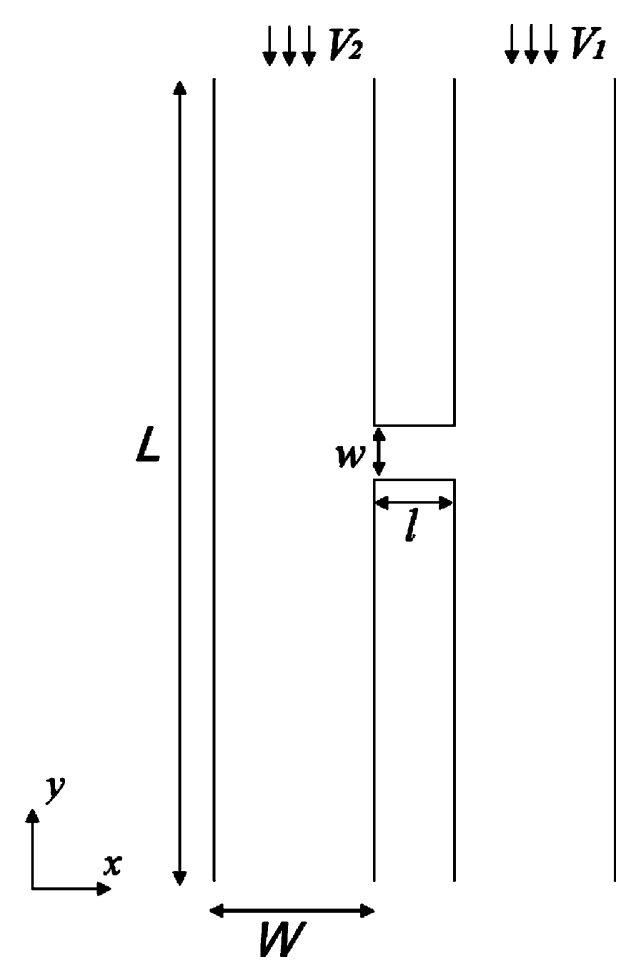

Fig. 1 Schematic of the microchannel coculture device (not drawn to scale). The right and left main chambers have the same width $W$ and length $L$. The connection channel has length $l$ and with $w$. Two perfusion velocities, $V_{1}$ and $V_{2}$, are applied at the inlets of two chambers, respectively. The origin of coordinates is located at the left bottom corner of the left chamber 
culture channel. $V_{1}$ and $V_{2}$ are the perfusion velocity at the inlet of the right and the left channels, respectively. Such configuration can be extended to have more than two channels and multiple connection channels. During the culture, the culture media is delivered into the two main channels at different flow rates. Since we will first examine the general picture for the transport of the growth factor without considering its consumption, a $2 \mathrm{D}$ approximation is reasonable because the variation in concentration across the channel depth can be neglected. Later when we simulate the monolayer cell growth and MTS growth, the 2D computational domain will be extended toward depth direction to form 3D geometry.

The mass transport of the growth factor in the microchannel bioreactor is governed by

$\frac{\partial C}{\partial t}+\vec{v} \cdot \nabla C=D \nabla^{2} C+R$

where $C$ is the concentration of the growth factor, $\vec{v}$ is the velocity vector, $D$ is the diffusion coefficient, and $R$ is the volumetric uptake rate for the growth factor. It should be noted that the term $R$ is taken into account only in the simulation of monolayer cell growth and MTS growth. This uptake rate follows the Michaelis-Menten (MM) kinetics (Michaelis and Menten 1913)

$R=\frac{V_{\max } C}{K_{m}+C}$

where $V_{\max }$ and $K_{m}$ are the MM parameters.

Since the concentration does not affect the flow field, the flow field $\vec{v}$ in the microchannel bioreactor can be described by the steady-state Navier-Stokes equation and the continuity equation:

$\rho \vec{v} \cdot \nabla \vec{v}=-\nabla P+\mu \nabla^{2} \vec{v}$

$\nabla \vec{v}=0$

where $\rho$ is the density of fluid, $P$ is the pressure, and $\mu$ is the viscosity of fluid.

For the monolayer culture, the cell proliferation in the microchannel is modeled by the continuous cell growth equation

$\frac{\partial \phi}{\partial t}=\mu_{g} \phi$

where $\phi$ represents the cell density and $\mu_{g}$ represents the specific growth rate of cell. Here the cell growth rate $\mu_{g}$ is assumed to depend only on the concentration of growth factor provided that all other nutrients (i.e., glucose and oxygen) are sufficient for cell growth. A logistic model (Pathi et al. 2005) is used to describe cell growth

$\mu_{g}=\mu_{\max }\left(1-\frac{\phi}{\phi_{\max }}\right) \mu_{\mathrm{GF}}$ where $\mu_{\max }$ is the maximal growth rate under optimized conditions, $\phi_{\max }$ is the maximal cell density supported by the bioreactor, and $\mu_{\mathrm{GF}}$ is the contribution of the growth factor.

Based on the experimental data (Reddy et al. 1994), we assume a logarithmically linear relationship between the scaled growth rate and the growth factor concentration

$\frac{\mu_{\mathrm{GF}}-\mu_{0}}{\mu_{\max }-\mu_{0}}=A \log 10\left(\frac{C}{C_{0}}\right)$

where $\mu_{0}$ is the control growth rate with a corresponding concentration $C_{0}$, and $A$ is the fitting coefficient determined from the experimental data. It should be noted that there exist minimal effective growth factor concentration and saturation concentration beyond which the growth rate is almost independent on the growth factor.

For the MTS growth, the volume change of the spherically symmetric MTS is given by

$\frac{\mathrm{d} V}{\mathrm{~d} t}=\mu_{g} V$.

There are few mathematical models directly relating the MTS growth rate to the growth factor. Therefore, we use the same formula, Eq. 6, to describe the MTS growth since the MTS volume is proportional to the total cell number without considering the internal tumor cell death. This assumption is valid for the early growth stage of the MTS.

For the simulation of cell or MTS growth, the mass transport Eq. 1 is coupled to the growth Eqs. 4 or 7 via the mass transfer flux at the monolayer-media interface for the monolayer culture or the flux at the tumor spheroid surface.

$-\vec{n} \cdot(D \nabla C)=F$

where flux term $F$ is obtained by multiplying the volumetric uptake rate, Eq. 2, by the cell density $\phi$ for the monolayer culture, or by multiplying Eq. 2 by the tumor spheroid volume (total cell number inside the spheroid) and then dividing by the surface area of the tumor spheroid for the MTS culture.

To provide more general guidance for the coculture bioreactor design, we use nondimensional parameters in the present study. One of the important nondimensional parameters is Peclet number that is a measure of the relative importance of convection to diffusion in the mass transfer (of the growth factor in this study),

$P e=\frac{\bar{U} W}{D}$

where $\bar{U}$ is the average velocity at the inlet of culture channel, $W$ is the main channel width, and $D$ is the diffusion coefficient. For a given molecule and the liquid medium, the higher the $P e$ number, the higher the flow (convection) velocity. The main channel width $W$ is also 
used to normalize other geometry parameters $L, l, w$ as shown in Fig. 1 and the MTS diameter. In addition, the time $t$ is normalized by the cell doubling time $T_{\mathrm{d}}$ and the concentration is normalized by the inlet concentration $C_{0}$.

All the equations are solved numerically using a finite element package Multiphysics V3.1 (Comsol AB Inc.). Since the concentration field does not affect the flow field, Navier-Stokes Eq. 3 only needs to be solved once for the given flow rate. For the simulation of the monolayer cell growth and the MTS growth, the mass transport Eq. 1 can be decoupled from the growth Eqs. 4 or 7 because the time to reach a steady state of concentration distribution is much shorter than the cell growth time scale, which significantly simplifies the numerical computations.

\section{Results and discussion}

\subsection{Transport of growth factor}

We first study the transport and the distribution of the growth factor in the coculture microchannel chip, without considering the growth of the monolayer cells or multicellular tumor spheroid (MTS) spheroid. Since the convection and the diffusion mainly occur in the channel width direction, a 2D model is sufficient for describing such cases as shown in Fig. 1.

To better mimic in vivo environment, continuous-flow perfusion bioreactors, especially microfluidic based devices, have been widely used in the cell-based biological research. In a static coculture environment of the conventional cell culture system, the communication between different cell types is realized solely by the diffusion of the signaling chemicals. However, the cell communications may have distinct characteristics in the continuous-flow bioreactor, mainly due to the effects of the perfusion velocity. Figure 2 shows the normalized concentration field of the growth factor within the microchannel coculture system where the same perfusion velocity is applied at the inlet of the right and the left culture channels. We assume that the growth factor is generated only by one type of cells in the right channel (i.e., the non-dimensional concentration is set to 1 at the inlet of the right channel). The concentration field of the growth factor for two $P e$ numbers $(P e=3,000$ and $P e=30$, corresponding to the real velocities $1 \mathrm{~mm} / \mathrm{s}$ and $10 \mu \mathrm{m} / \mathrm{s}$ provided that the main channel width is $W=300 \mu \mathrm{m}$ and the diffusion coefficient of the growth factor is $D=1 \times 10^{-10} \mathrm{~m}^{2} / \mathrm{s}$ ) was studied for different lengths of the connection channels $(l=W, w=$ $0.1 W$ in Fig. 2a and $l=0.4 W, w=0.1 W$ in Fig. 2b). It is found that almost no growth factor can reach the left channel although there is some diffusion through the connection channel. Because the same perfusion velocity is (a)

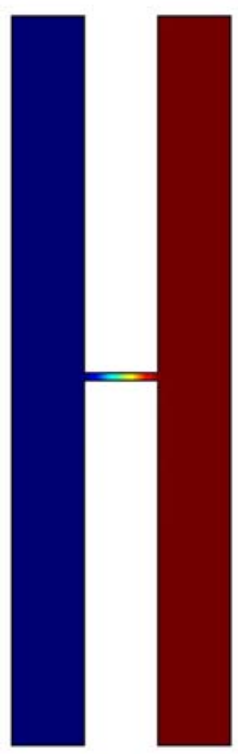

$P e=3000$

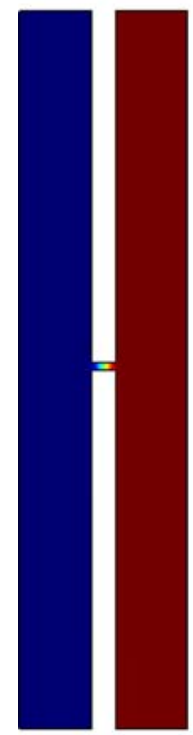

$P e=3000$

(b)

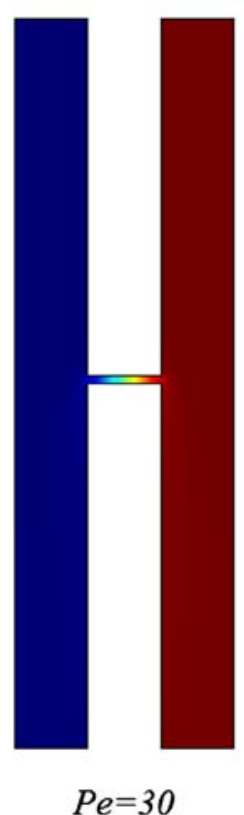

$\operatorname{Max} 1.0$
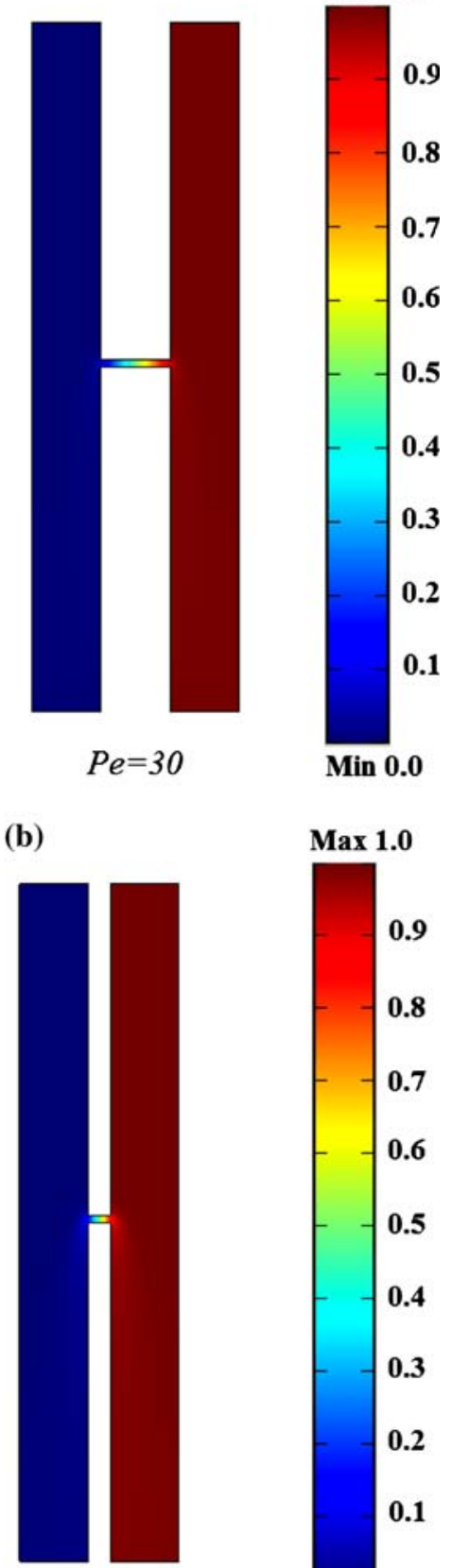

$P e=30$
$\operatorname{Max} 1.0$

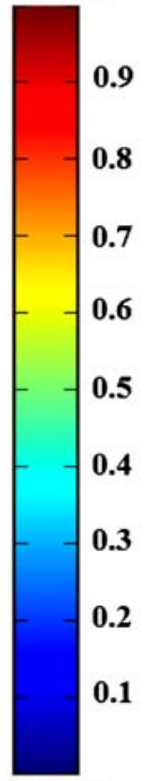

Min 0.0

Fig. 2 Normalized concentration fields of the growth factor in the devices with different lengths of the connection channel under the same perfusion velocities $(P e=3,000$ and $P e=30)$. a $l=W, w=$ $0.1 \mathrm{~W} ; \mathbf{b} l=0.4 \mathrm{~W}, w=0.1 \mathrm{~W}$. The color scale bar, as well as those in Figs. 3b, 4a, 6a, and 7, indicates the normalized growth factor concentration that is scaled by the inlet concentration

applied at the inlet of the right and the left culture channels, hence no pressure difference exists across the connection channel, and the motion of the growth factor from the right to the left relies only on the diffusion. Due to the convection effect, the growth factor is washed away toward the 
outlet of the left channel before it can be diffused into the major part of the left channel. The poor communication is not improved even if very low perfusion velocity and short connection channel are used (Fig. 2b). Therefore, in a coculture system with the parallel microchannels using the same perfusion velocity, different cell types in separate channels cannot communicate with each other effectively because the convection effects suppress the diffusion effects.

As discussed above, in order to facilitate the communication between different cells in the parallel microchannels, two different perfusion velocities must be applied at inlets of the left and the right channels. Due to the velocity difference, as shown in Fig. 3a, a transverse flow will be generated within the connection channel from the right main channel $(P e=3,000)$ to the left one $(P e=30)$ and thus greatly enhance the transport of the growth factor between two channels. Figure $3 \mathrm{~b}$ displays the concentration field of the growth factor. It can be clearly seen that the growth factor flows into the left channel from the right channel via the connection channel. Unlike mutual signaling effects in the static coculture system where the signaling chemicals from all cell types will finally diffuse everywhere, the communications between different cells in the present continuous-flow perfusion bioreactor occur in a unidirectional way. The signaling chemicals can only be delivered from the cells in the high velocity area to the cells in the low velocity area. This mechanism can be utilized to actively control the signaling processes between cells. For example, by adjusting the velocity difference between the right and the left channels, the amount of signaling chemicals transported from one side to the other can be adjusted; by increasing the perfusion velocity in the left channel and decreasing the perfusion velocity in the right channel, the transport of the signaling chemicals and thus the cellular communications can be reversed.

In the coculture chip with a fixed main channel width, the transport of the signaling chemicals depends on the width and length of the connection channel because the hydraulic resistance of the channel is directly related to the channel length. Under the same perfusion velocities applied in the right and the left channels, smaller hydraulic resistance allows higher transverse convection in the connection channel and thus more transport of the chemicals. Figure $4 \mathrm{a}$ shows the comparison of the concentration fields of the growth factor for varied connection channels. As expected, the shorter and the wider the connection channel is, the better the growth factor is delivered from one channel to the other channel. Figure $4 \mathrm{~b}$ plots the concentration distributions along the left channel width at the downstream position $y=2.5 \mathrm{~W}$ measured from the channel outlet. The shortest and widest connection channel $(l=$ $0.2 W, w=0.2 W$ ) has the most uniform distribution of the

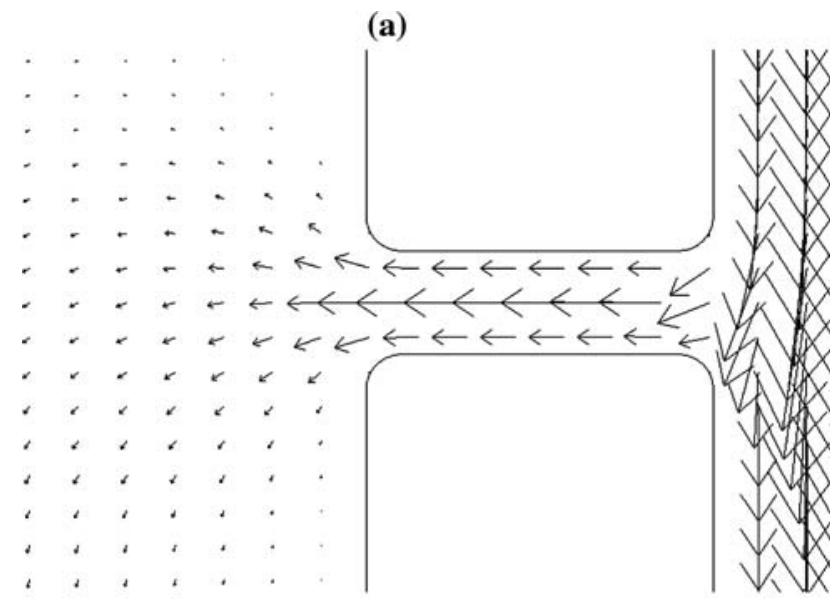

(b)

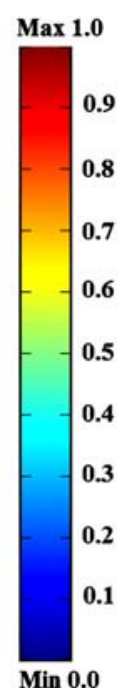

Fig. 3 a Velocity vector around the connection channel; b normalized concentration field of the growth factor. Different perfusion velocities $(P e=3,000$ and $P e=30)$ applied at the inlets of the right chamber and left chamber

growth factor across the channel width. Moreover, it is found that increasing the width of the connection channel is more effective than decreasing the length to better deliver the growth factor. Figure 5 shows the concentration distributions along the channel width at three different downstream positions $y=0, y=2 W$, and $y=4 W$ for this geometry. With the increasing distance toward the downstream, the concentration becomes more uniform because of the diffusion effect. Therefore, cells at different position are exposed to concentration gradients of the signaling chemicals and may display heterogeneous pattern during growth. We will demonstrate these phenomena later.

To obtain more uniform concentration of the growth factor in the left channel, it is necessary to use wide connection channel between culture channels. However, the cells may also migrate or be washed from one channel to another channel if the connection channel is too big. Instead of a single wide connection channel, an alternative 
(a)

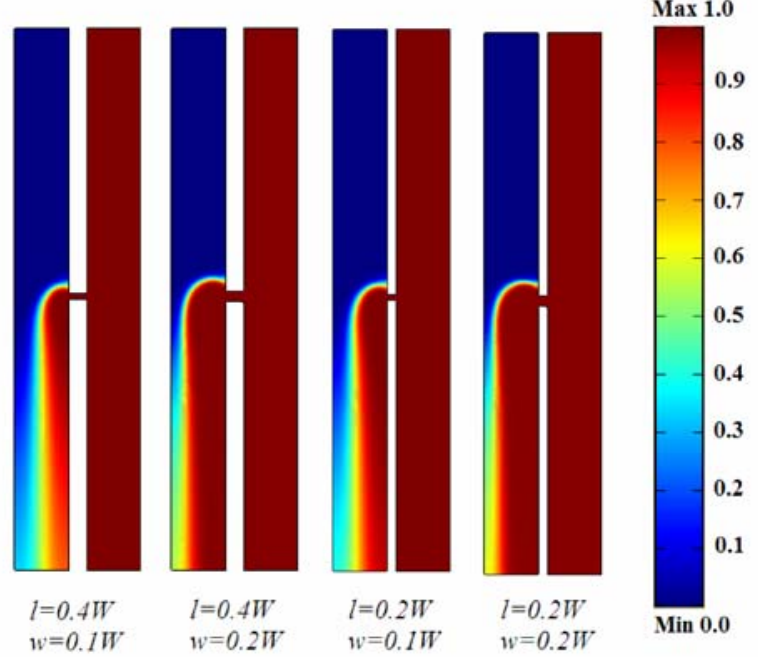

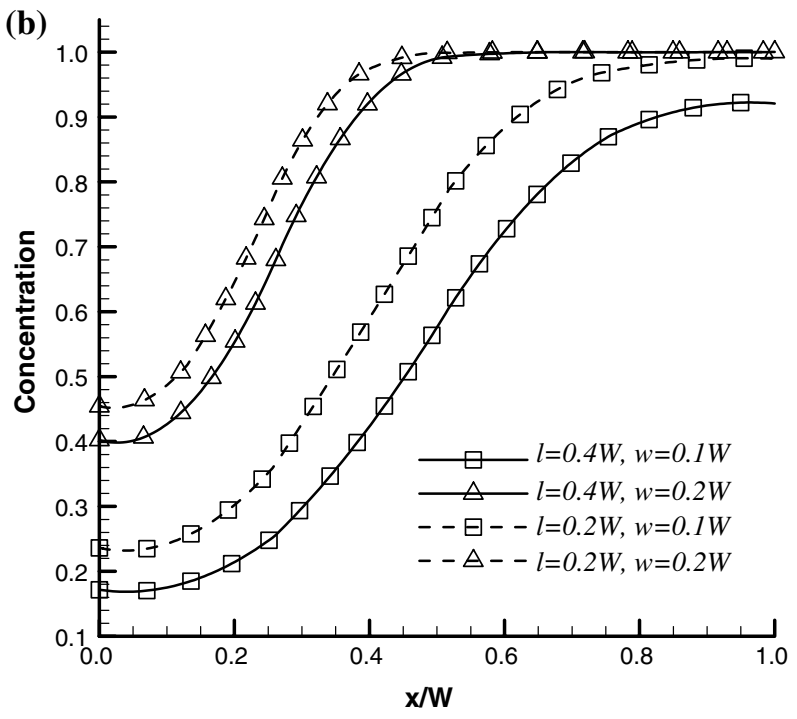

Fig. 4 Comparison of the transport of the growth factor in the coculture devices with different connection chambers. a Normalized concentration field; $\mathbf{b}$ normalized concentration distribution along the left chamber width at $y=2.5 \mathrm{~W}$

way is to use multiple narrow connection channels that can deliver more uniform signaling chemicals while still separate different cell types. Figure 6a shows the concentration field in a coculture system with multiple connection channels. Three connection channels, located at $y=5 \mathrm{~W}$, $5.3 \mathrm{~W}$, and $5.6 \mathrm{~W}$, have same geometry of $l=0.2 \mathrm{~W}$ and $w=0.1 W$. Compared to the previous concentration field resulting from the single channel (Fig. 4a), using multiple connection channels significantly improves the delivery of the growth factor. Figure $6 \mathrm{~b}$ compares the concentration distributions along the channel width for single and multiple connections and clearly shows that the distributions in the multiple channels are more uniform.

It is desirable to have the capability to study the signaling process among several different cell types using one

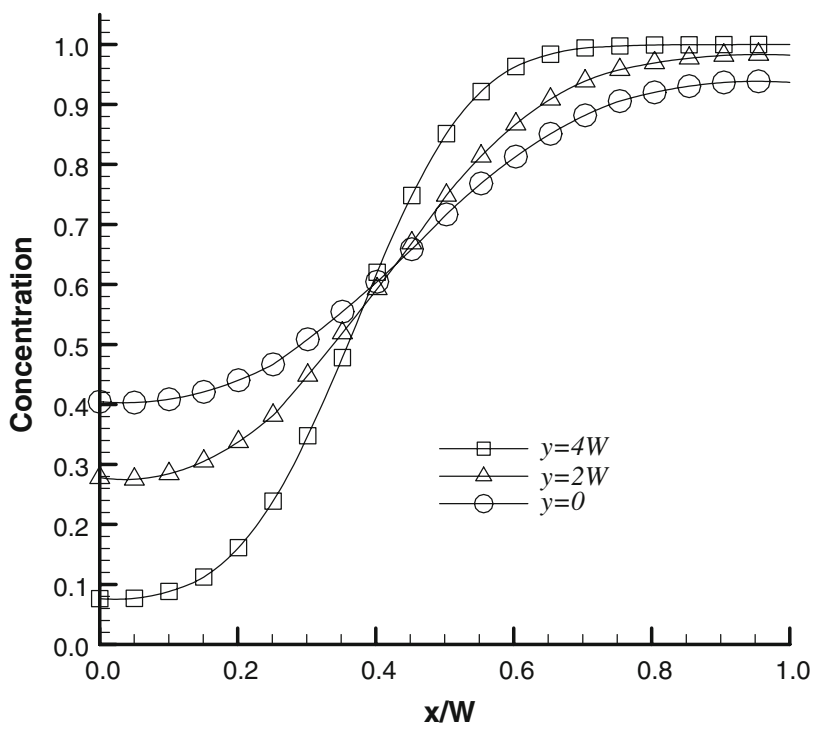

Fig. 5 Comparison of the normalized concentration distribution along the left chamber width at different downstream positions for the connection channel of $l=0.2 \mathrm{~W}, w=0.2 \mathrm{~W}$

coculture chip at the same time. The two-channel chip described above can be extended to realize such a function by simply adding more parallel culture channels. The findings mentioned above are still valid in the cases of multiple channels. By adjusting the perfusion velocities at the different channels, we can actively control the direction and the extent of signaling process. Figure 7 represents a potential configuration of the continuous-flow coculture chip for multiple cell types. In Fig. 7a, a high perfusion velocity $(P e=3,000)$ is applied at the middle channel while same low velocity $(\mathrm{Pe}=30)$ is applied at the right and left channel. Therefore, the signaling chemicals generated by the cells in the middle channel will be evenly delivered into different cell types in the side channels. On the other hand, it is also possible to study how the presence of multiple cell types affects one cell type by applying high perfusion velocities at the right and the left channels and low velocity at the middle channel. Figure $7 \mathrm{~b}$ shows the concentration field using such velocity combination $(P e=3,000$ at the right and the left channels and $P e=30$ at the middle channel, respectively). The growth factors from both side channels flow into the middle channel and thus regulate the growth of the cells in the middle channel.

\subsection{Monolayer cell growth}

As described in the preceding section, the growth factor delivered from one channel into another channel in the continuous-flow bioreactor will form concentration gradients and thus result in a heterogeneous growth of cells. We simulated the transient process of cell growth in the case of 
(a)

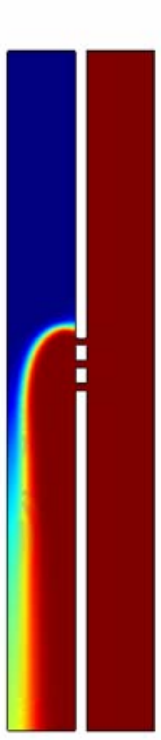

$\operatorname{Max} 1.0$
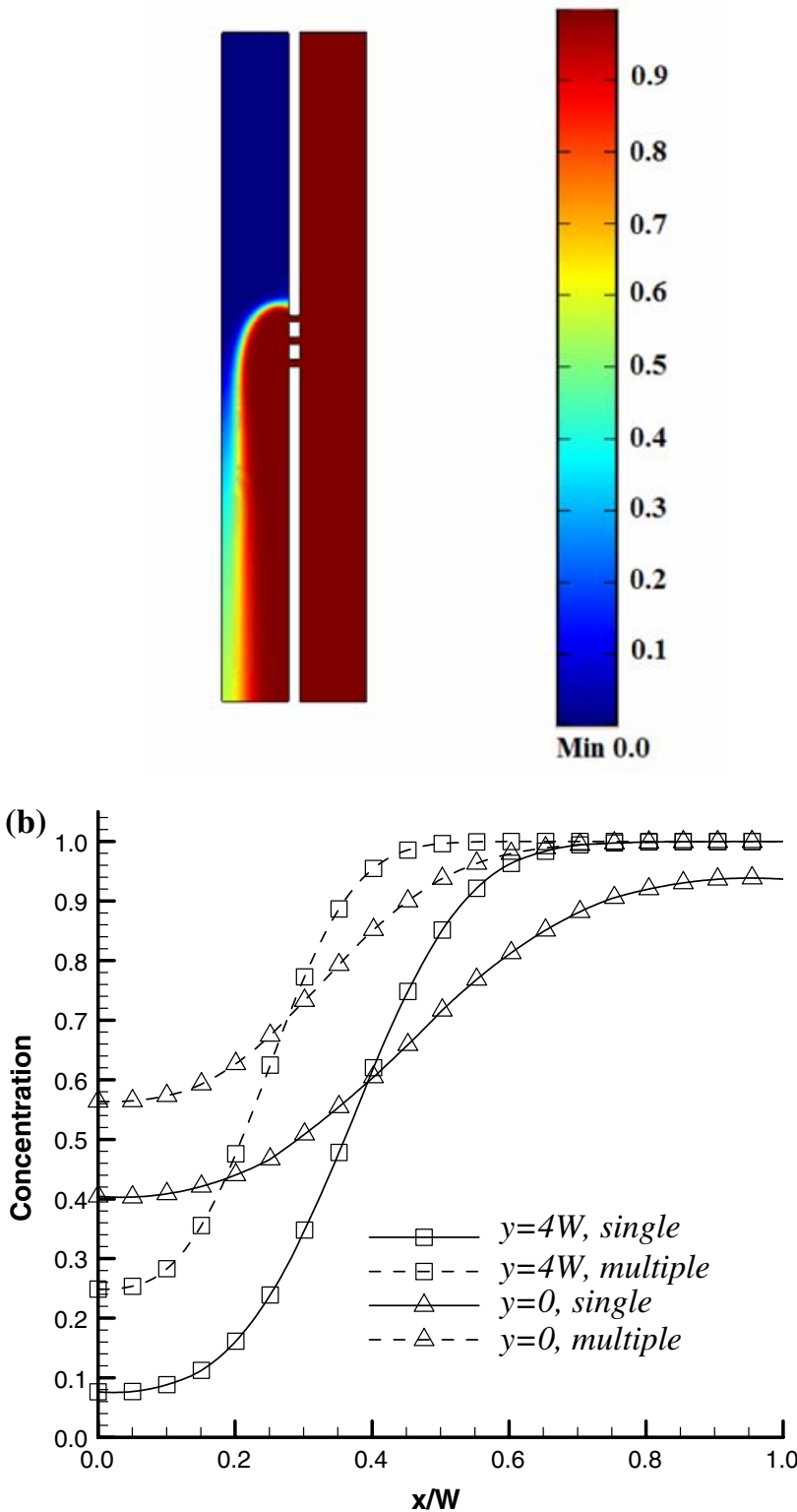

Fig. 6 Transport of the growth factor using multiple connection channels. a Concentration field; b comparison of the normalized concentration distributions along the left chamber width for single and multiple connections

the monolayer culture. Other nutrients (for example, oxygen and glucose) and signaling chemicals are assumed be sufficient so that the cell growth rate only depends on the concentration of the growth factor. The multiple connection 2D channel used in the Fig. 6a is extruded with a height of $W$ in Z-direction to form a 3D microchannel coculture system. Initially, monolayer cells are seeded at the channel bottom of the downstream part of the culture channel with a cell density $\phi_{0}$. A high perfusion velocity $P e=3,000$ is applied at the right channel while a low velocity $P e=30$ is applied at the left channel. Figure 8a displays the variation of the cell (a)

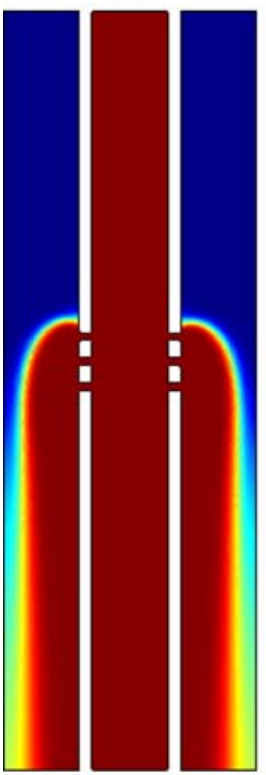

(b)

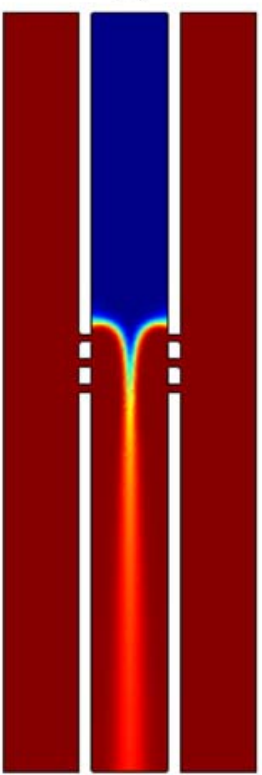

$\operatorname{Max} 1.0$

Min 0.0

Fig. 7 Normalized concentration fields in the multiple culture chambers. a High velocity $(P e=3,000)$ at the middle chamber and low velocity $(P e=30)$ at the side chambers; $\mathbf{b}$ a cascade of velocities $(P e=3,000,300$, and 30) from the right to the left

density at the bottom after $10 T_{\mathrm{d}}$. Here the cell density is normalized by the initial cell density $\phi_{0}$. Figure $8 \mathrm{~b}$ depicts the cell density along the channel width at the middle point of the downstream channel. To show the relative variation, the cell density is normalized by the local maximal density $\phi_{\max }$. It is found that even using the multiple connection channels to facilitate the transport of the growth factor, the cell density still could have $30 \%$ difference in the width direction.

\subsection{MTS growth}

Although cancers are extremely diverse, a multicellular tumor spheroid (MTS) can be used as to capture the most relevant features of tumor growth kinetics, in both experimental investigation and theoretical study. Because the tumor consumes more nutrients than normal cells (Gatenby 1996), it is expected that the concentration gradients in the continuous-flow coculture environment will affect the MTS growth. We used the same chip and in Sect 3.2. The tumor spheroid is fixed at the bottom of the left channel, as shown by the small 3D schematics in Fig. 9a. As shown in Fig. 5, the concentration across the width direction varies with the different downstream locations. The MTS exposed to more growth factor will grow faster. We calculated the growth of two MTSs that are fixed at different downstream positions $(y=3.5 \mathrm{~W}$ and $y=1.5 \mathrm{~W})$. Figure $9 \mathrm{a}$ displays the normalized 
(a)

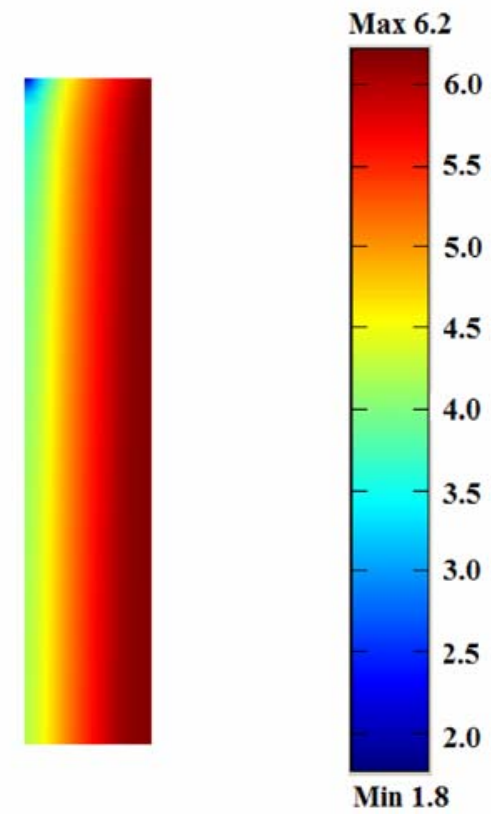

(b)

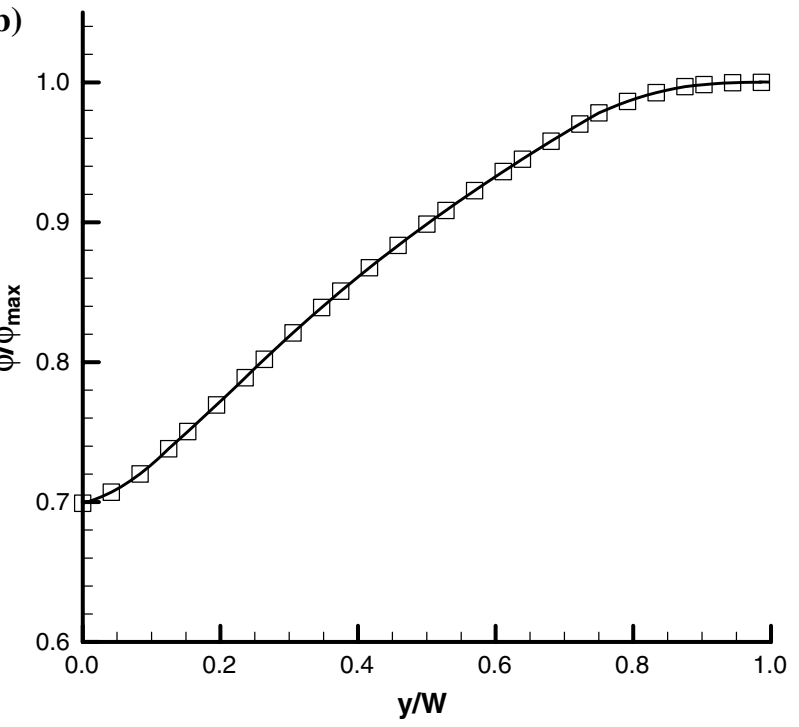

Fig. 8 Cell density variations of monolayer culture caused by the growth factor concentration gradient. a Cell density at the bottom of the left chamber; $\mathbf{b}$ cell density variation along the left chamber width at $y=2.5 \mathrm{~W}$. The cell density is normalized by the initial cell density

concentration field of the growth factor around the MTS at different positions. To get a better view for the 3D field, we plot the concentrations at the central $y-z$ plane $(x=0.5 W)$ of the left channel and at the outer surface of the tumor spheroid. Figure 9a clearly shows that the concentration at the outer surface of the MTS becomes more uniform with the increase of the distance toward downstream. At $y=$ $3.5 \mathrm{~W}$, the minimal surface concentration of the growth factor is only about 0.4 while it increases to about 0.75 at $y=$ $1.5 \mathrm{~W}$. The growth rate of the MTS can be estimated by Eq. 6 using the averaged concentration over the outer (a)
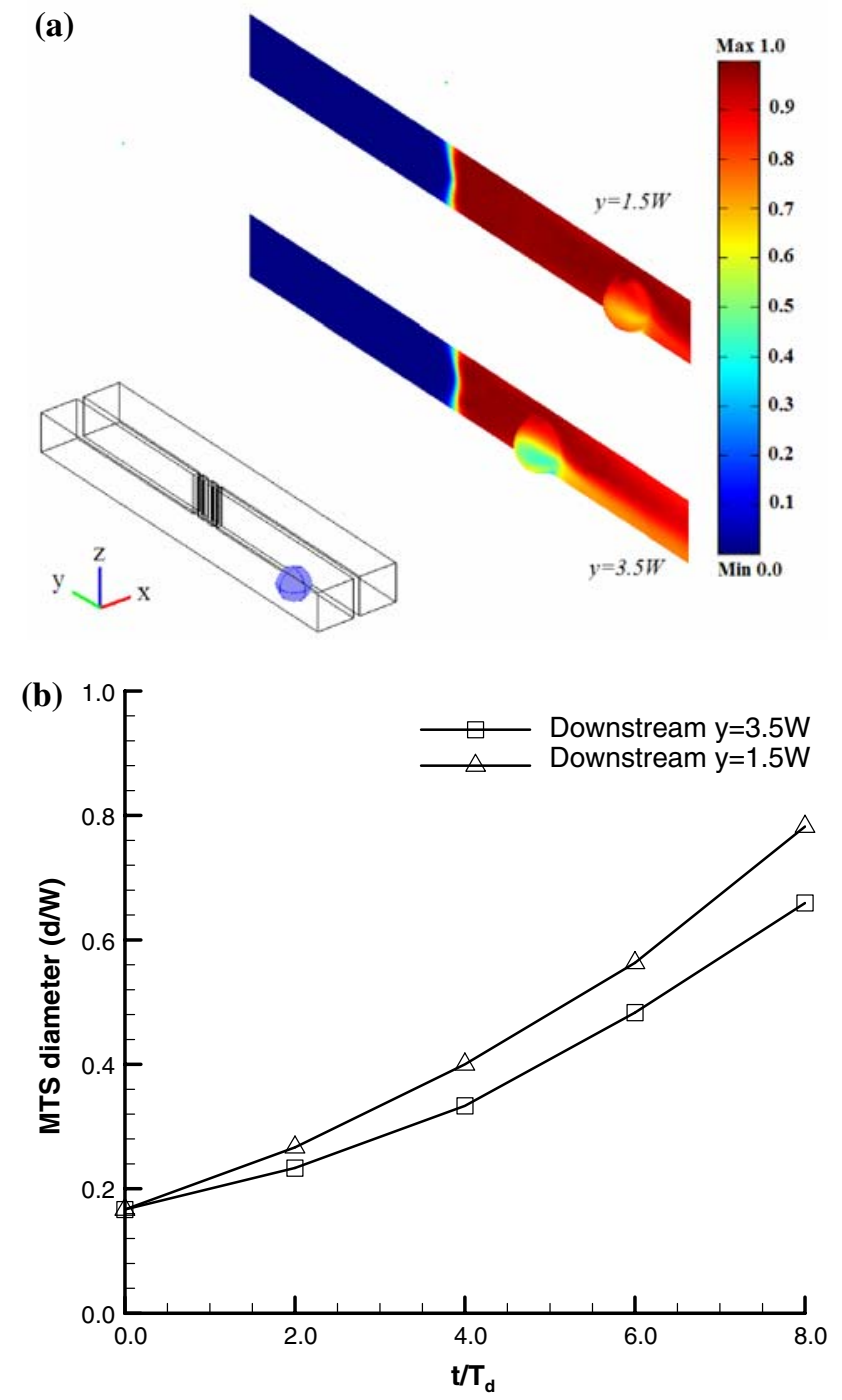

Fig. 9 The growth of MTSs in the coculture device. a Normalized concentration fields of the growth factor at the central plane of the left chamber and outer surface of the MTS; $\mathbf{b}$ comparison of the change in diameter of MTSs grown at different downstream positions

surface. Figure $9 \mathrm{~b}$ shows the comparison of the change of the MTS diameters at these two positions. Due to the low growth factor concentration, the growth of the MTS at $y=$ $3.5 \mathrm{~W}$ lags behind that at $y=1.5 \mathrm{~W}$. Such growth difference caused by the concentration gradients should be taken into account in designing the continuous-flow perfusion bioreactor for the MTS coculture.

\section{Conclusions}

We have developed a theoretical model to investigate the transport of the signaling chemicals in the microchannel coculture chips. Using an EGF-like growth factor as the sample chemical, the effects of velocities and channel 
geometry were studied for the continuous-flow bioreactors. It is found that different perfusion velocities must be applied in the parallel channels to facilitate the signaling communication between cells. Such communications occur in a unidirectional way since the signaling chemicals can only flow from the high velocity area to the low velocity area. It is, however, possible to actively reverse and control the communication direction by adjusting the perfusion velocities during coculture. The growth of the monolayer cells and the MTS in the continuous-flow coculture environment were also simulated using 3D models. The numerical results confirmed that the concentration gradients will induce the heterogeneous growth of the cells and the MTSs, which should be taken into account in designing the continuousflow perfusion bioreactor for the cell coculture research.

Acknowledgments The authors are grateful to the support from the School of Engineering, Vanderbilt University, and from the H. Fort Flowers Foundation.

\section{References}

Anderson H, van den Berg A (2004) Microfabrication and microfluidics for tissue engineering: state of the art and future opportunities. Lab Chip 4:98-103

Attawia MA, Nicholson JJ, Laurencin CT (1999) Coculture system to assess biocompatibility of candidate orthopaedic materials. Clin Orthop 365:230-236

El-Ali J, Sorger PK, Jensen KF (2006) Cells on chips. Nature 442:403-411

Ellington JE, Carney EW, Farrell PB, Simkin ME, Foote RH (1990) Bovine 1-2 cell embryo development using a simple medium in three oviduct epithelial cell coculture systems. Biol Reprod 43:97-104

Fillinger MF, O'Connor SE, Wagner RJ, Cronenwett JL (1993) The effect of endothelial cell coculture on smooth muscle cell proliferation. J Vasc Surg 17:1058-1067

Finch PW, Rubin JS, Miki T, Ron D, Aaronson SA (1989) Human KGF is FGF-related with properties of a paracrine effector of epithelial cell growth. Science 245:752-755

Folch A, Toner M (1998) Cellular micropatterns on biocompatible materials. Biotechnol Prog 14:388-392

Gatenby RA (1996) Altered glucose metabolism and the invasive tumor phenotype: insights provided through mathematical models. Int J Oncol 8:597-601

Hu G, Li D (2007) Three-dimensional modeling of transport of nutrients for multicellular tumor spheroid culture in a microchannel. Biomed Microdevices 9:315-323

Hu G, Quaranta V, Li D (2007) Modeling of effects of nutrient gradients on cell proliferation in microfluidic bioreactor. Biotechnol Prog 23:1347-1354

Irimia D, Charras G, Agrawal N, Mitchison T, Toner M (2007) Polar stimulation and constrained cell migration in microfluidic channels. Lab Chip 7:1791-1799

Kane BJ, Zinner MJ, Yarmush ML, Toner M (2006) Liver-specific functional studies in a microfluidic array of primary mammalian hepatocytes. Anal Chem 78:4291-4298

Klein B, Zhang XG, Jourdan M, Content J, Houssiau F, Aarden L, Piechaczyk M, Bataille R (1989) Paracrine rather than autocrine regulation of myeloma-cell growth and differentiation by interleukin-6. Blood 73:517-526
Lauffenburger DA, Forsten KE, Will B, Wiley HS (1995) Molecular/ cell engineering approach to autocrine ligand control of cell function. Ann Biomed Eng 23:208-215

Leclerc E, Sakai Y, Fujii T (2003) Cell culture in 3-dimensional microfluidic structure of PDMS (polydimethylsiloxane). Biomed Microdevices 5:109-114

Martinoia S, Bove M, Tedesco M, Margesin B, Grattarola M (1999) A simple microfluidic system for patterning populations of neurons on silicon micromachined substrates. J Neurosci Methods 87:3544

Mehta K, Linderman J (2006) Model-based analysis and design of a microchannel reactor for tissue engineering. Biotechnol Bioeng 94:596-609

Michaelis M, Menten ML (1913) Kinetics of invertase action. Z Biochem 49:333-369

Pantoja R, Nagarah JM, Starace DM, Melosh NA, Blunck R, Bezanilla F, Heath JR (2004) Silicon chip-based patch-clamp electrodes integrated with PDMS microfluidics. Biosens Bioelectron 20:509-517

Pathi P, Ma T, Locke BR (2005) Role of nutrient supply on cell growth in bioreactor design for tissue engineering of hematopoietic cells. Biotechnol Bioeng 89:743-758

Powers CJ, McLeskey SW, Wellstein A (2000) Fibroblast growth factors, their receptors and signaling. Endocrine-Related Cancer 7:165-197

Powers MJ, Domansky K, Kaazempur-Mofrad MR, Kalezi A, Capitano A, Upadhyaya A, Kurzawski P, Wack KE, Stolz DB, Kamm R, Griffith LG (2002) A microfabricated array bioreactor for perfused 3D liver culture. Biotechnol Bioeng 78:257-269

Ray LB (2007) Enabling endocrine action of fibroblast growth factors. Sci STKE 382:tw133

Reddy CC, Wells A, Lauffenburger DA (1994) Proliferative response of fibroblasts expressing internalization-deficient epidermal growth-factor (EGF) receptors is altered via differential EGF depletion effect. Biotechnol Prog 10:377-384

Rhee SW, Taylor AM, Tu CH, Cribbs DH, Cotman CW, Jeon NL (2005) Patterned cell culture inside microfluidic devices. Lab Chip 5:102-107

Roy P, Baskaran H, Tiles AW, Yarmush ML, Toner M (2001) Analysis of oxygen transport to hepatocytes in a flat-plate microchannel bioreactor. Ann Biomed Eng 29:947-955

Sporn MB, Todaro GJ (1980) Autocrine secretion and malignant transformation of cells. N Engl J Med 303:878-880

Tan W, Desai TA (2004) Microscale multilayer coculture for biommimetic blood vessels. J Biomed Mater Res A 72A:146-160

Taylor AM, Blurton-Jones M, Rhee SW, Cribbs DH, Cotman CW, Jeon NL (2005) A microfluidic culture platform for CNS axonal injury, regeneration and transport. Nat Methods 2:599-605

Thio CL, Waxman SG, Sontheimer H (1993) Ion channels in spinal cord astrocytes in vitro III. Moduclation of channel expression by coculture with neurons and neuron-conditioned medium. J Neurophysiol 69:819-831

Tourovskaia A, Figueroa-Masot X, Folch A (2005) Differentiationon-a-chip: a microfluidic platform for long-term cell culture studies. Lab Chip 5:14-19

Walker GM, Ozers MS, Beebe DJ (2002) Insect cell culture in microfluidic channels. Biomed Microdevices 4:161-166

Walker GM, Zeringue HC, Beebe DJ (2004) Microenvironment design considerations for cellular studies. Lab Chip 4:91-97

Wei CW, Cheng JY, Young TH (2006) Elucidating in vitro cell-cell interaction using a microfluidic coculture system. Biomed Microdevices 8:65-71

Williams KA, Saini S, Wick TM (2002) Computational fluid dynamics modeling of steady-state momentum and mass transport in a bioreactor for cartilage tissue engineering. Biotechnol Prog 18:951-963 